\title{
Job stress is associated with migraine in current workers: The Brazilian Longitudinal Study of Adult Health (ELSA-Brasil)
}

\author{
I.S. Santos ${ }^{1,2}$, R.H. Griep ${ }^{3}$, M.G.M. Alves ${ }^{4}$, A.C. Goulart ${ }^{2}$, P.A. Lotufo ${ }^{1,2}$, S.M. Barreto ${ }^{5}$, D. Chor $^{6}$, \\ I.M. Benseñor ${ }^{1,2}$ \\ 1 Departamento de Clínica Médica, Faculdade de Medicina da Universidade de São Paulo, Brazil \\ 2 Centre for Clinical and Epidemiological Research, Hospital Universitário da Universidade de São Paulo, Brazil \\ 3 Laboratory of Health and Environment Education, Fundação Oswaldo Cruz, Rio de Janeiro, Brazil \\ 4 Departamento de Planejamento em Saúde do Instituto de Saúde da Comunidade, Universidade Federal Fluminense, Rio de Janeiro, Brazil \\ 5 Faculty of Medicine, Universidade Federal de Minas Gerais, Belo Horizonte, Brazil \\ 6 Escola Nacional de Saúde Pública, Fundação Oswaldo Cruz, Rio de Janeiro, Brazil
}

\begin{abstract}
Correspondence
Itamar S. Santos

E-mail: itamarss@usp.br

\section{Funding sources}

The ELSA-Brasil baseline study was supported by the Brazilian Ministry of Health (Science and Technology Department) and the Brazilian Ministry of Science and Technology (Financiadora de Estudos e Projetos and CNPq National Research Council) (grants 01 060010.00 RS, 01060212.00 BA, 0106 0300.00 ES, $01060278.00 \mathrm{MG}, 0106$ $0115.00 \mathrm{SP}, 01060071.00 \mathrm{RJ})$. The funding source did not contribute to study design, data analysis or decision to publish.
\end{abstract}

Conflicts of interest

The authors report no conflicts of interest. As a disclosure, R.H.G., P.A.L., S.M.B., D.C. and I.M.B. are recipients of a fellowship from the Brazilian National Research Council (CNPq).

Accepted for publication

18 February 2014

doi:10.1002/j.1532-2149.2014.489.x

\begin{abstract}
Background: Migraine is an important source of social burden and work-related costs. Studies addressing the association of migraine with job stress are rare.

Objectives: The aim of this paper was to study the association of job stress components and migraine using structured, validated questionnaires that were part of the Brazilian Longitudinal Study of Adult Health (ELSA-Brasil). Methods: The ELSA-Brasil is a multicentre cohort of 15,105 civil servants $(12,096$ current workers) in Brazil. Job strain was assessed using the 17-item Brazilian version of the Swedish Demand-Control-Support Questionnaire. Headache episodes in the preceding 12 months were assessed using a questionnaire based on the International Headache Society criteria. We analysed the association between job stress domains and migraine in men and women using adjusted logistic regression and interaction models.

Results: We included 3113 individuals without headache and 3259 migraineurs. Low job control [odds ratio (OR) 1.30; 95\% confidence interval $(95 \%$ CI) $1.10-1.53$, high job demands (OR 1.37; 95\% CI 1.18-1.59) and low social support (OR 1.49; 95\% CI 1.29-1.71) were associated with migraine. Job control was more strongly associated with migraine in women ( $p$ for interaction $=0.02$ ). High-strain (high demand and low control) jobs were associated with migraine in both men (OR 1.48; 95\% CI 1.11-1.97) and women (OR 1.51; 95\% CI 1.17-1.95).

Conclusions: We observed a strong association between high-strain jobs and migraine. Job control was a stronger migraine-related factor for women. Low social support was associated with migraine in both sexes.
\end{abstract}

\section{Background}

Migraine is an important source of personal burden (Hasselström et al., 2002) and worsens the quality of life (Lipton et al., 2003). Migraine also produces a marked social load (Stewart et al., 2003), with estimated costs of more than $€ 27$ billion and US\$13 billion to Europe and the United States each year, respectively. Indirect work-related costs (absenteeism and loss of productivity) are responsible for more than $90 \%$ of this amount (Hu et al., 1999; Andlin-Sobocki et al., 2005; Hawkins et al., 2007). Work is a substantial source of stress for many individuals. Previous studies have demonstrated that stressful jobs predict several health-related outcomes, including cardiovascular diseases (Kivimäki et al., 2012), musculoskeletal 


\section{What's already known about this topic?}

- Stress is a commonly cited trigger for migraine.

- However, existing data regarding the role of work stress components in migraine yield conflicting results.

\section{What does this study add?}

- Using a structured, consistent methodology, we found a strong association between high-strain jobs and migraine.

- Job control was a stronger migraine-related factor for women.

- Low job social support was associated with migraine in both sexes.

pain (Haukka et al., 2011), absenteeism (Griep et al., 2010) and loss of productive time (Macedo et al., 2007). The most widely used tool for assessing stress related to work characteristics is based on Karasek's demand/control model (Karasek, 1979; Lopes et al., 2010). The perception of social support was later developed as an additional tool (Johnson and Hall, 1988).

Some studies have focused on sex differences in the role of job stress as a health determinant. A large meta-analytic study by Kivimäki et al. (2012) evaluated the effect of job stress on the risk of coronary heart disease. In that study, although job strain was significantly associated with coronary heart disease, no sexspecific differences were observed. Rivera-Torres et al. (2013) recently described differences between men and women in work-related stress and its relationship to health risk perceptions. The authors found that lower job support was associated with worse risk perception in both sexes, but this effect was more prominent in women. In addition, intellectually and emotionally demanding jobs were associated with worse risk perception in women, but not in men. It is not possible, however, to extend those results to specific health conditions.

Little is known regarding the role of work stress in migraine, and existing data yield conflicting results. Wilkins and Beaudet (1998) analysed cross-sectional data from the National Population Health Survey in Canada. In that study, they found an association between previous migraine diagnosis and high job strain and low co-worker support in men. For women, no such associations were found. In the largest prospective study to date, Mäki et al. (2007) did not find an association between new-onset migraine and job strain in women (as measured by Karasek's model).
Although that study had a large sample, the authors focused on new-onset cases, which may limit the possible conclusions concerning the association between migraine and job stress. Two-thirds of that study sample was 40 years old or older, and incident migraine typically occurs at younger ages (Stewart et al., 2008).

The aim of the present article is to study the association between job strain and migraine in current workers who participated in the baseline assessments of the Brazilian Longitudinal Study of Adult Health (ELSA-Brasil). As previous studies have identified sex differences in the association of specific job stress domains with health outcomes, we also hypothesized that job demand, control and social support could be heterogeneously associated with migraine in men and women.

\section{Methods}

\subsection{Study design}

The ELSA-Brasil design and concepts have been detailed elsewhere (Aquino et al., 2012). Briefly, ELSA-Brasil is a cohort study of 15,105 civil servants (12,096 current workers and 3009 retired workers) from five universities and one research institute located in six cities (São Paulo, Belo Horizonte, Porto Alegre, Salvador, Rio de Janeiro and Vitoria). The job characteristics among these institutions vary greatly. Among ELSA-Brasil participants, there are nonskilled manual workers, clerical workers and professionals, including health care workers and faculty.

Baseline assessments took place from August 2008 to December 2010 and included in-person interviews conducted by trained personnel. These interviews focused on socio-demographic characteristics, health and medical histories, occupational exposure, family history of disease, health care, psychosocial factors, food consumption, smoking, alcohol consumption, physical activity and medication use. Clinical and laboratory measurements were also performed.

\subsection{Measures}

\subsubsection{Job stress}

Job demands, job control, and social support were evaluated using the 17-item Brazilian version (Alves et al., 2004) of the Swedish Demand-Control-Support Questionnaire (DCSQ), which is based on the Job Content Questionnaire (JCQ) (Karasek, 1979). The JCQ considers the influence and the interrelationship of two components in the work process: (1) psychological demand - work overload, degree of difficulty, available time, time allotted to execute tasks and the existence of contradictory or discordant orders and (2) control autonomy and skills relating to the process, which are 
required from the worker. Johnson and Hall (1988) included the perception of social support in the model. Social support involves social and emotional integration, trust and assistance in performing tasks in the work environment.

Similar to the JCQ, the DCSQ presents items related to psychological job demands and decision latitude. The job demand subscale includes four questions measuring time and speed for performing tasks and one question assessing conflicts between different demands. The job control subscale includes four questions related to the use and development of abilities and two related to decision latitude in relation to the work process. Social support at work subscale includes six questions regarding the feedback and support from colleagues and supervisors. (Table 1). All responses were provided along a 4-point Likert-type scale. The Brazilian version of DCSQ has been determined to have intraclass correlation coefficients of $0.88,0.87$ and 0.86 and Cronbach's alpha coefficients of $0.72,0.63$ and 0.86 for the demand, control and social support domains, respectively (Alves et al., 2004). In addition, the adequacy of the dimensional structure of this instrument in Brazil was demonstrated in two recently published articles (Griep et al., 2009; Hökerberg et al., 2010).

The ELSA-Brasil participants were classified in groups of similar size, within each dimension. Therefore, control (score range 6 to 24 points), demand (score range 5 to 20 points) and support (score range 6 to 24 points) scores were classified as 'low', 'medium' or 'high'. In accordance with Kara-

Table 1 Short version of the job stress scale (Alves et al., 2004).

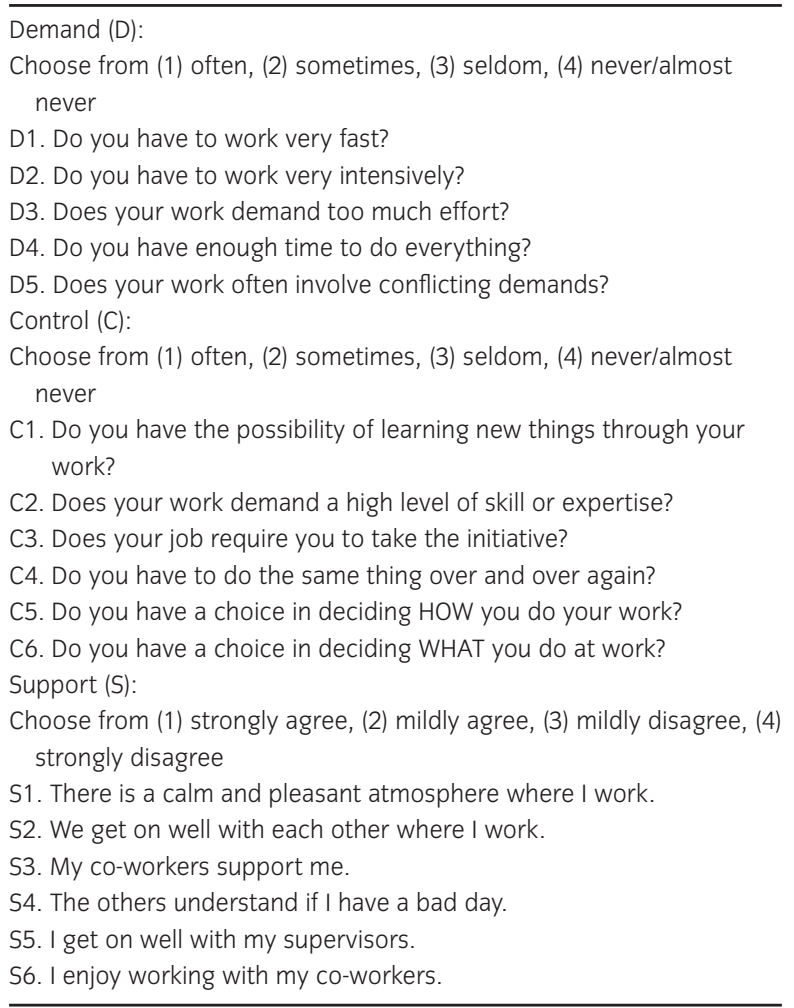

sek's model, job demands and job control in the ELSA-Brasil were dichotomized as low (up to median value) or high (above median value), and the participants were allocated into four quadrants to express the relationship between job demand/control: (1) low-strain work (low demand/high control), (2) passive (low demand/low control), (3) active (high demand/high control) and (4) high-strain work (high demand/low control) (Karasek, 1979).

\subsubsection{Headache questionnaire and migraine diagnosis}

All participants who answered 'yes' to the question 'in the last 12 months, have you had a headache?' at the ELSABrasil baseline evaluation were invited to answer a detailed questionnaire based on the International Headache Society (IHS) criteria (The Headache Classification Subcommittee of the International Headache Society, 2004). This questionnaire has been translated into Brazilian Portuguese, validated (Benseñor et al., 1997; Jardim et al., 2001) and used in previous studies (Benseñor et al., 2008, 2011). This questionnaire assesses pain frequency, duration, quality, location, intensity, triggering factors and accompanying symptoms, such as nausea or vomiting and the presence of aura. Migraine was defined as the presence of criteria for IHS codes 1.1 (migraine without aura) or 1.2 (migraine with aura), with the exception of episode duration. Because use of medication for migraine treatment is typical in the country without medical prescription (Benseñor, 2001; Loyola Filho et al., 2002), we did not include episode duration as part of the criteria, which is consistent with definitions adopted elsewhere (Lipton et al., 2001; Diamond et al., 2007). Compared with a migraine diagnosis established by a specialist's consultation, the Brazilian version of this questionnaire had a kappa coefficient agreement of $0.58,78.3 \%$ sensitivity, $83.3 \%$ specificity, $90.4 \%$ positive predictive value and $65.8 \%$ negative predictive value. (Jardim et al., 2001).

For this paper, individuals with headache in the previous year who failed to fulfill one (reference IHS code 1.6, probable migraine) or more migraine criteria (other headaches) were not included in analyses.

\subsubsection{Other variables}

Race was self-defined as Black, Brown (Mixed), White, Asian or Native. For this study, the Asian and Native categories were grouped as 'others' because the number of individuals in these categories was too small. Age was classified in strata by 10 -year intervals (35-44, 45-54, 55-64, 65-74). Participants reported net family income and educational level, and we stratified these variables accordingly. Local currency [Brazilian reais (BRL)] was converted to U.S dollars (USD) at a rate of BRL 2.00 = USD 1.00.

\subsection{Statistical analysis}

Chi-square tests were performed whenever applicable. The Wilcoxon rank sum test was used for age comparisons 
between subgroups. Within the group of current workers, we generated binary logistic regression models, adjusted for age, sex, education level and race, to identify whether job demands, job control and social support (either as categorical or continuous variables) were associated with migraine. Data were also analysed after being stratified by sex and adjusted for the other variables.

In addition, we analysed the association between job classification (according to Karasek's model) and migraine. We evaluated the association of passive, active and high-strain work with migraine, using low-strain work as the reference level in a crude model and two multivariate models (multivariate model 1 : adjusted for sex, age, income, race and occupational level; model 2: all variables in multivariate model 1 plus social support). These models were generated separately for men and women. Interaction regression models were created to analyse if the associations between job domains or job classification according to Karasek's model with migraine were influenced by sex. Analyses were performed using R for Windows version x 64 2.15.1 (R Core Team, 2012) and the epicalc package (Chongsuvivatwong, 2012). A significance level of 0.05 was considered in all analyses.

\subsection{Ethical considerations}

The study protocol is in accordance with the Helsinki Declaration of 1975, as revised in 1983. Approvals from all institutional review boards were granted, and all individuals signed informed consent forms.

\section{Results}

Of 12,096 current workers in the ELSA-Brasil baseline assessment, $289(2.4 \%)$ had missing answers that precluded headache classification, and 5391 (44.6\%) had headache episodes that did not meet IHS criteria for migraine with or without aura. Of the remaining 6416 subjects, $44(0.7 \%)$ were excluded because of missing information on job stress scales. The remaining 6372 individuals (3113 without headache and 3259 with migraine) were included in the analyses. Table 2 presents the bivariate analysis of socio-demographic information for current workers in the ELSA-Brasil using headache classification and job stress quadrants from Karasek's model as grouping variables.

Individuals with migraine more frequently had high-demand, low-control jobs as compared with the no headache group (Table 3 ). Low social support and high-strain jobs (according to Karasek's model) were also more common among migraineurs than among respondents without headache.

Fully adjusted regression models indicated positive, independent associations between migraine and high job demands, low job control and low social support, after adjustment for age, sex, income, educational level and race (Table 4). Analysing job domains as continuous variables, we also found a significant positive association between migraine and job demand score $(p<0.01)$ and a significant negative association between migraine and both job control $(p=0.01)$ and social support scores $(p<0.01)$ in the fully adjusted models.

Sex-specific analyses revealed a more consistent association between low job control and migraine in women compared with men. In fact, the interaction models indicated a significant influence of sex on the association between low job control and migraine $(p=0.02)$. When analysed as a continuous variable, job control scores were still associated with migraine for women $(p=0.02)$ but not for men $(p=0.18)$. All other associations between job strain scores (as continuous variables) and migraine remained significant in sex-specific analyses.

We also analysed data according to job strain quadrants using Karasek's demand/control model (Table 5). Considering men and women together, active and high-strain jobs were positively associated with migraine compared with low-strain work in all models. In sex-specific analyses, high-strain jobs (for both men and women) and active jobs (for men) were consistently associated with migraine in all models.

\section{Discussion}

We observed a strong association between job stress and migraine in the ELSA-Brasil cohort baseline assessment. There were also sex-specific differences in the association of migraine and job strain domains, as migraine exhibited a stronger association with job control in women. Moreover, when analysing job demands and control using Karasek's job strain model, individuals with passive, active or high-strain work were significantly more likely to have a diagnosis of migraine compared with participants with low-strain work.

Stress is a commonly cited trigger for migraine episodes (Henry et al., 2002). In a study of 1750 migraine patients in the United States, $75.9 \%$ reported the presence of triggering factors. Within this group, stress was reported by $79.7 \%$ (Kelman, 2007). Stress frequency has also been related to migraine activity, and a there is a significant temporal correlation between the occurrence of stressor events and migraine episodes (Holm et al., 1997; Sauro and Becker, 2009).

The association between stress and migraine and the high burden and costs involved make the study of the association of migraine and job conditions a subject of 
Table 2 ELSA-Brasil current workers according to headache and job stress classification.

\begin{tabular}{|c|c|c|c|c|c|c|c|}
\hline & \multicolumn{2}{|c|}{ Headache classification } & \multicolumn{4}{|c|}{ Job stress by Karasek's model } & \multirow[b]{2}{*}{$\begin{array}{l}\text { Total } \\
n=6372\end{array}$} \\
\hline & $\begin{array}{l}\text { No headache } \\
n=3113\end{array}$ & $\begin{array}{l}\text { Migraine } \\
n=3259\end{array}$ & $\begin{array}{l}\text { Low strain } \\
n=1590\end{array}$ & $\begin{array}{l}\text { Passive } \\
n=2405\end{array}$ & $\begin{array}{l}\text { Active } \\
n=1162\end{array}$ & $\begin{array}{l}\text { High strain } \\
n=1215\end{array}$ & \\
\hline \multicolumn{8}{|l|}{ Age (years) } \\
\hline Median & 52.0 & $48.0^{*}$ & 50.0 & $49.0^{\star \star \star \star}$ & $49.0 * \star \star$ & $48.0 * \star \star$ & 49.0 \\
\hline Interquartile range & $46.0-57.0$ & $43.0-52.0$ & $45.0-56.0$ & $45.0-55.0$ & $45.0-55.0$ & $44.0-53.0$ & $44.0-55.0$ \\
\hline \multicolumn{8}{|l|}{ Age strata } \\
\hline $35-44$ years & $566(18.2)$ & $1036(31.8)^{\star}$ & $371(23.2)$ & $573(23.8)^{\star \star \star}$ & $290(25.0)^{\star \star \star}$ & $368(30.3)^{\star \star \star}$ & $1602(25.1)$ \\
\hline $45-54$ years & $1391(44.7)$ & $1667(51.2)$ & $693(43.6)$ & $1204(50.1)$ & $559(48.1)$ & $602(49.5)$ & $3058(48.0)$ \\
\hline 55-64 years & $1025(32.9)$ & $531(16.3)$ & $460(28.9)$ & $577(24.0)$ & $288(24.8)$ & $231(19.0)$ & $1556(24.4)$ \\
\hline $65-74$ years & $131(4.2)$ & $25(0.8)$ & $66(4.2)$ & $51(2.1)$ & $25(2.2)$ & $14(1.2)$ & $156(2.4)$ \\
\hline \multicolumn{8}{|l|}{ Sex } \\
\hline Female & 997 (32.0) & $2489(76.4)^{\star}$ & $738(46.4)$ & $1303(54.2)^{\star \star \star}$ & $645(55.5)^{\star \star \star}$ & $800(65.8)^{\star \star \star *}$ & $3486(54.7)$ \\
\hline Male & $2116(68.0)$ & $770(23.6)$ & $852(53.6)$ & $1102(45.8)$ & $517(44.5)$ & 415 (34.2) & $2886(45.3)$ \\
\hline \multicolumn{8}{|l|}{ Race } \\
\hline White & $1421(46.4)$ & $1606(49.6)^{\star *}$ & $895(57.2)$ & $946(39.8)^{\star \star \star}$ & $692(60.4)$ & $494(40.9)^{\star \star \star}$ & $3027(48.1)$ \\
\hline Brown & $956(31.2)$ & $966(29.9)$ & $394(25.2)$ & $842(35.4)$ & $275(24.0)$ & $411(34.1)$ & $1922(30.5)$ \\
\hline Black & $561(18.3)$ & $560(17.3)$ & 214 (13.7) & $510(21.4)$ & $137(12.0)$ & $260(21.5)$ & $1121(17.8)$ \\
\hline Other & $123(4.0)$ & $104(3.2)$ & $62(4.0)$ & $81(3.4)$ & $42(3.7)$ & $42(3.5)$ & $228(3.6)$ \\
\hline \multicolumn{8}{|l|}{ Family income } \\
\hline$\leq$ USD 1244 & $978(31.5)$ & $943(29.0)^{\star}$ & $262(16.5)$ & $1025(42.8)^{\star \star \star}$ & $154(13.3)^{\star \star \star \star}$ & $480(39.6)^{\star \star \star}$ & $1921(30.2$ \\
\hline USD 1245-3319 & $1281(41.3)$ & $1558(47.9)$ & $662(41.7)$ & $1121(46.8)$ & $472(40.7)$ & $584(48.2)$ & $2839(44.7)$ \\
\hline$\geq$ USD 3320 & $842(27.2)$ & $750(23.1)$ & $662(41.7)$ & $248(10.4)$ & $534(46.0)$ & $148(12.2)$ & $1592(25.1)$ \\
\hline \multicolumn{8}{|l|}{ Educational level } \\
\hline Lower than High school & $494(15.9)$ & $294(9.0)^{*}$ & $104(6.6)$ & $472(19.6)^{\star \star \star}$ & $60(5.2)^{\star \star \star}$ & $152(12.5)^{\star \star \star}$ & $788(12.4)$ \\
\hline High school & $1142(36.7)$ & $1324(40.6)$ & $402(25.3)$ & $1225(50.9)$ & $227(19.5)$ & $612(50.4)$ & 2466 (38.7) \\
\hline College or higher & $1477(47.4)$ & $1641(50.4)$ & $1084(68.2)$ & $708(29.4)$ & 875 (75.3) & $451(37.1)$ & $3118(48.9)$ \\
\hline
\end{tabular}

Age strata, sex, race, family income, and educational level expressed as count and column percentages. ELSA-Brasil, Brazilian Longitudinal Study of Adult Health; USD, U.S. dollars.

${ }^{*} p<0.01$ compared with no headache subgroup.

$\star * p<0.05$ compared with no headache subgroup

$* * * p<0.01$ compared with low-strain subgroup.

$\star * * * p<0.05$ compared with low-strain subgroup.

the utmost importance. The aforementioned study by Mäki et al. (2007) is representative of the limited available data regarding this association (Mäki et al., 2007). Our results conflict with the findings of the previous study; however, there are differences in the study designs. In the Finnish study, migraine diagnoses were assessed exclusively by auto-referral, asking if any doctor had ever told the patient he or she had migraine. There is evidence, however, that this approach has a low sensitivity, as a substantial number of individuals with migraine remains undiagnosed. Lipton et al. (1992) analysed data from 20,468 subjects and determined that only $41 \%$ of female and $29 \%$ of male migraineurs (defined using a symptom-based questionnaire) reported a migraine diagnosis made by a physician. In the present work, we assessed symptoms of migraine in the preceding 12 months, regardless of previous diagnosis, using a structured and validated questionnaire, and we administered these questionnaires in person with trained staff to avoid this bias.
Table 3 Job stress domains and job stress classification using Karasek's model in individuals without headache and migraine.

\begin{tabular}{|c|c|c|c|}
\hline & $\begin{array}{l}\text { No headache } \\
n=3113\end{array}$ & $\begin{array}{l}\text { Migraine } \\
n=3259\end{array}$ & $p$ \\
\hline Job demand (score range) & & & $<0.01$ \\
\hline Low (s11 points) & $907(29.1)$ & $736(22.6)$ & \\
\hline Medium (12 to 14 points) & $1203(38.6)$ & $1149(35.3)$ & \\
\hline High ( $\geq 15$ points) & $1003(32.2)$ & $1374(42.2)$ & \\
\hline Job control (score range) & & & $<0.01$ \\
\hline Low (s16 points) & $952(30.6)$ & $1071(32.9)$ & \\
\hline Medium (17 to 19 points) & $1080(34.7)$ & $1345(41.3)$ & \\
\hline High ( $\geq 20$ points) & $1081(34.7)$ & $843(25.9)$ & \\
\hline Social support (score range) & & & $<0.01$ \\
\hline Low (s18 points) & $861(27.7)$ & $1228(37.7)$ & \\
\hline Medium (19 to 21 points) & $973(31.3)$ & $1091(33.5)$ & \\
\hline High ( $\geq 22$ points) & $1279(41.1)$ & $940(28.8)$ & \\
\hline $\begin{array}{l}\text { Job classification according to } \\
\text { Karasek's model }\end{array}$ & & & $<0.01$ \\
\hline Low strain & $903(29.0)$ & $687(21.1)$ & \\
\hline Passive & $1207(38.8)$ & $1198(36.8)$ & \\
\hline Active & $544(17.5)$ & $618(19.0)$ & \\
\hline High strain & 459 (14.7) & 756 (23.2) & \\
\hline
\end{tabular}


Table 4 Odds ratios of job strain domain groups for migraine according to sex after adjustment for age, income, race and educational level.

\begin{tabular}{|c|c|c|c|c|c|}
\hline \multirow{2}{*}{\multicolumn{2}{|c|}{ Adjusted or for migraine }} & \multirow[b]{2}{*}{ All } & \multicolumn{2}{|l|}{ Stratified by sex } & \multirow[b]{2}{*}{$p$ for interaction with sex } \\
\hline & & & Men & Women & \\
\hline \multirow[t]{5}{*}{ Job control } & High & 1.0 (Reference) & 1.0 (Reference) & 1.0 (Reference) & \\
\hline & Medium & $1.38(1.19-1.60)$ & $1.30(1.05-1.61)$ & $1.44(1.18-1.77)$ & 0.18 \\
\hline & & $p<0.01$ & $p=0.02$ & $p<0.01$ & \\
\hline & Low & $1.30(1.10-1.53)$ & $1.13(0.88-1.45)$ & $1.43(1.14-1.79)$ & 0.02 \\
\hline & & $p<0.01$ & $p=0.32$ & $p<0.01$ & \\
\hline \multirow[t]{5}{*}{ Job demand } & Low & 1.0 (Reference) & 1.0 (Reference) & 1.0 (Reference) & \\
\hline & Medium & $1.06(0.91-1.23)$ & $1.04(0.84-1.29)$ & $1.10(0.90-1.35)$ & 0.62 \\
\hline & & $p=0.45$ & $p=0.74$ & $p=0.36$ & \\
\hline & High & $1.37(1.18-1.59)$ & $1.40(1.13-1.74)$ & $1.37(1.12-1.68)$ & 0.88 \\
\hline & & $p<0.01$ & $p<0.01$ & $p<0.01$ & \\
\hline \multirow[t]{5}{*}{ Social support } & High & 1.0 (Reference) & 1.0 (Reference) & 1.0 (Reference) & \\
\hline & Medium & $1.29(1.12-1.49)$ & $1.37(1.12-1.69)$ & $1.22(1.01-1.49)$ & 0.84 \\
\hline & & $p<0.01$ & $p<0.01$ & $p=0.04$ & \\
\hline & Low & $1.49(1.29-1.71)$ & $1.57(1.27-1.94)$ & $1.44(1.19-1.75)$ & 0.58 \\
\hline & & $p<0.01$ & $p<0.01$ & $p<0.01$ & \\
\hline
\end{tabular}

Second, the target of the previous study was new-onset migraine. Approximately two-thirds of these researchers' cohort was older than 40 years. Although migraine episodes may still occur (Misakian et al., 2003), newonset migraine is not as common at this age; therefore, this subset most likely does not represent the typical individual with migraine. In fact, the number of sub- jects excluded at baseline because of previous migraine diagnosis in that study was more than seven times the number of new-onset cases (8998 vs. 1281). It is reasonable to expect that in the ELSA-Brasil, most of the migraineurs also became symptomatic several years before assessment. Thus, we assessed prevalent (not incident) migraine symptoms in a similar age strata,

Table 5 Crude and adjusted odds ratios for migraine in ELSA-Brasil current workers according to Karasek's job strain quadrants and stratified by sex.

\begin{tabular}{|c|c|c|c|c|c|}
\hline \multirow[b]{2}{*}{ Adjusted or for migraine } & & \multirow[b]{2}{*}{ All } & \multicolumn{2}{|l|}{ Stratified by sex } & \multirow[b]{2}{*}{$p$ for interaction with sex } \\
\hline & & & Men & Women & \\
\hline \multirow[t]{7}{*}{ Age- and sex-adjusted } & Low strain & 1.0 (Reference) & 1.0 (Reference) & 1.0 (Reference) & \\
\hline & Passive & $1.13(0.97-1.31)$ & $1.09(0.88-1.35)$ & $1.19(0.97-1.46)$ & 0.49 \\
\hline & & $p=0.11$ & $p=0.42$ & $p=0.10$ & \\
\hline & Active & $1.30(1.09-1.55)$ & $1.43(1.11-1.83)$ & $1.22(0.96-1.55)$ & 0.43 \\
\hline & & $p<0.01$ & $p<0.01$ & $p=0.10$ & \\
\hline & High strain & $1.54(1.29-1.83)$ & $1.49(1.15-1.94)$ & $1.59(1.25-2.01)$ & 0.56 \\
\hline & & $p<0.01$ & $p<0.01$ & $p<0.01$ & \\
\hline \multirow[t]{7}{*}{ Multivariate model 1} & Low strain & 1.0 (Reference) & 1.0 (Reference) & 1.0 (Reference) & \\
\hline & Passive & $1.19(1.01-1.39)$ & $1.18(0.94-1.49)$ & $1.21(0.97-1.51)$ & 0.36 \\
\hline & & $p=0.03$ & $p=0.16$ & $p=0.09$ & \\
\hline & Active & $1.31(1.10-1.56)$ & $1.41(1.09-1.81)$ & $1.25(0.98-1.60)$ & 0.54 \\
\hline & & $p<0.01$ & $p<0.01$ & $p=0.07$ & \\
\hline & High strain & $1.63(1.36-1.96)$ & $1.61(1.22-2.13)$ & $1.63(1.27-2.10)$ & 0.46 \\
\hline & & $p<0.01$ & $p<0.01$ & $p<0.01$ & \\
\hline \multirow[t]{7}{*}{ Multivariate model 2} & Low strain & 1.0 (Reference) & 1.0 (Reference) & 1.0 (Reference) & \\
\hline & Passive & $1.16(0.99-1.35)$ & $1.16(0.92-1.46)$ & $1.17(0.94-1.46)$ & 0.41 \\
\hline & & $p=0.07$ & $p=0.22$ & $p=0.16$ & \\
\hline & Active & $1.24(1.04-1.48)$ & $1.33(1.03-1.71)$ & $1.19(0.93-1.52)$ & 0.50 \\
\hline & & $p=0.02$ & $p=0.03$ & $p=0.18$ & \\
\hline & High strain & $1.50(1.25-1.81)$ & $1.48(1.11-1.97)$ & $1.51(1.17-1.95)$ & 0.46 \\
\hline & & $p<0.01$ & $p<0.01$ & $p<0.01$ & \\
\hline
\end{tabular}

Multivariate model 1 is adjusted for sex, age, income, race and educational level. Multivariate model 2 is adjusted for sex, age, income, race, educational level and social support. ELSA-Brasil, Brazilian Longitudinal Study of Adult Health. 
targeting most of the productive lifetime. These differences may explain, at least partially, these researchers' negative results in contrast with our positive results. Kopec and Sayre (2004) analysed the longitudinal data of 6062 participants of the National Population Health Survey in Canada between 1994/1995 and 1998/1999. They found an association between any new-onset pain and/or discomfort and high work stress in that study [odds ratio (OR) 1.80; 95\% confidence interval (CI) 1.28-2.52] but not between new-onset migraine and high-stress work (OR 1.03; 95\% CI 0.56-1.89). Similar to the study by Mäki et al. (2007), the number of individuals excluded because of previous diagnosis was higher than the number of new-onset migraine cases (503 vs. 275).

Propensity for migraine is clearly different according to sex, as were the associations between job control and migraine in our study. Consistent with our results, Rivera-Torres et al. (2013) also found that lower social support was related to worse health risk perceptions in both genders. However, they observed this association was stronger in women, and we did not observe any such association. In addition, those researchers did not find a sex-related difference in response to job control (contrary to our results), and job demands were different between sexes only when qualitative subdomains (intellectually and emotionally demanding jobs) were analysed. These mixed results may be related to which dependent variables were chosen to model the influence of job strain on health. For these examples, it is reasonable to conclude that the occurrence of migraine, coronary disease risk and perceptions of health may not be equitably affected by job strain domains. From this point of view, sex-specific differences among these models may also be expected and should be studied separately for each model. In addition, in the Spanish study, data were not adjusted for other variables, such as occupational level and income, which may influence the association between migraine and job stress.

Our study has several strengths. It is the first study to date to assess the association between job stress and migraine using validated questionnaires, based on the IHS criteria and the Swedish DCSQ. This approach avoids bias due to underdiagnosing, a frequent scenario for migraine patients, and adds substantial and consistent information to the available published data. All interviews were conducted in person by trained staff, which guaranteed a notably high rate of response. The ELSA-Brasil participants are workers from five universities and one research institute in the country. The work characteristics within each of these facilities are typically highly diverse, which creates a favourable scenario for the study of work stress effects. The study, however, also has limitations. Data were obtained with a cross-sectional design, which does not allow for an inference about causality. It is noteworthy that, to date, prospective studies have not confirmed these associations. However, in addition to relying exclusively on auto-referred diagnosis, these longitudinal studies may be targeting a specific subset of migraine patients, instead of the whole group, as peak incidence of this condition occurs in earlier ages. Strong evidence supporting this interpretation of data is that the number of excluded individuals because of migraine diagnosis at baseline in these studies is substantially higher than the number of new-onset cases. An additional limitation to our study is that we may have missed some migraine diagnoses in individuals whose symptoms started at an earlier age and remitted before assessment. Considering this scenario, however, our results still may indicate an association between higher job stress and persistent migraine symptoms.

In addition to confirming the association of migraine and job stress components with a well-structured study design, our results indicate that there is rationale for future longitudinal (observational or interventional) studies in the workplace. These studies should not be restricted to new-onset cases. Rather, they should target the remission of symptoms in individuals with migraine, which is a much larger group of individuals. This may potentially reduce the disabilities and workrelated costs associated with this condition.

\section{Conclusions}

We observed consistent associations between job stress domains and migraine in middle-aged current civil servants in Brazil. High-strain jobs were independently associated with migraine. Low job control was a strong sex-specific factor associated with migraine in women. Low social support was associated with migraine in both sexes.

\section{Author contributions}

I.S.S. participated in the study design and statistical analysis and wrote the paper. R.H.G. and I.M.B. participated in the study design and data collection and helped to write the paper. M.G.M.A., A.C.G., P.A.L., S.M.B. and D.C. participated in the study design and data collection and contributed in the intellectual content of the paper.

\section{Acknowledgements}

The authors thank the ELSA-Brasil participants who agreed to collaborate in this study. We also thank the research team of the ELSA-Brasil study for their contributions. 


\section{References}

Alves, M.G.M., Chor, D., Faerstein, E., Lopes, C.S., Werneck, G.L. (2004). Short version of the 'job stress scale': A Portuguese-language adaptation. Rev Saude Publica 38, 1-7.

Andlin-Sobocki, P., Jönsson, B., Wittchen, H.U., Olesen, J. (2005). Cost of disorders of the brain in Europe. Eur J Neurol 12, 1-27.

Aquino, E.M., Barreto, S.M., Benseñor, I.M., Carvalho, M.S., Chor, D., Duncan, B.B., Lotufo, P.A., Mill, J.G., Molina Mdel, C., Mota, E.L., Passos, V.M., Schmidt, M.I., Szklo, M. (2012). Brazilian Longitudinal Study of Adult Health (ELSA-Brasil): Objectives and design. Am J Epidemiol 175, 315-324.

Benseñor, I.M. (2001). To use or not to use dipyrone? Or maybe, Central Station versus ER? That is the question. Sao Paulo Med J 119, 190-191.

Benseñor, I.M., Goulart, A.C., Lotufo, P.A., Menezes, P.R., Scazufca, M. (2011). Cardiovascular risk factors associated with migraine among the elderly with a low income: The Sao Paulo Ageing \& Health Study (SPAH). Cephalalgia 31, 331-337.

Benseñor, I.M., Lotufo, P.A., Goulart, A.C., Menezes, P.R., Scazufca, M. (2008). The prevalence of headache among elderly in a low-income area of São Paulo, Brazil. Cephalalgia 28, 329-333.

Benseñor, I.M., Lotufo, P.A., Pereira, A.C., Tannuri, A.C., Issa, F.K., Akashi, D., Fucciolo, D.Q., Kakuda, E.S., Kanashiro, H., Lobato, M.L., Titan, S.O., Galvão, T.F., Martins, M.A. (1997). Validation of a questionnaire for the diagnosis of headache in an outpatient clinic at a university hospital. Arq Neuropsiquiatr 55, 364-369, (in Portuguese).

Chongsuvivatwong, V. (2012). Epicalc: Epidemiological calculator. R package version 2.14.1.6.

Diamond, S., Bigal, M.E., Silberstein, S., Loder, E., Reed, M., Lipton, R.B. (2007). Patterns of diagnosis and acute and preventive treatment for migraine in the United States: Results from the American Migraine Prevalence and Prevention Study. Headache 47, 355-363.

Griep, R.H., Rotenberg, L., Chor, D., Toivanen, S., Landsbergis, P. (2010). Beyond simple approaches to studying the association between work characteristics and absenteeism: Combining the DCS and ERI models. Work Stress 24, 179-195.

Griep, R.H., Rotenberg, L., Vasconcellos, A.G., Landsbergis, P., Comaru, C.M., Alves, M.G. (2009). The psychometric properties of demandcontrol and effort-reward imbalance scales among Brazilian nurses. Int Arch Occup Environ Health 82, 1163-1172.

Hasselström, J., Liu-Palmgren, J., Rasjö-Wrååk, G. (2002). Prevalence of pain in general practice. Eur J Pain 6, 375-385.

Haukka, E., Leino-Arjas, P., Ojajärvi, A., Takala, E.-P., Viikari-Juntura, E., Riihimäki, H. (2011). Mental stress and psychosocial factors at work in relation to multiple-site musculoskeletal pain: A longitudinal study of kitchen workers. Eur J Pain 15, 432-438.

Hawkins, K., Wang, S., Rupnow, M.F. (2007). Indirect cost burden of migraine in the United States. J Occup Environ Med 49, 368-374.

Henry, P., Auray, J.P., Gaudin, A.F., Dartigues, J.F., Duru, G., Lantéri-Minet, M., Lucas, C., Pradalier, A., Chazot, G., El Hasnaoui, A. (2002). Prevalence and clinical characteristics of migraine in France. Neurology 59, 232-237.

Holm, J.E., Lokken, C., Myers, T.C. (1997). Migraine and stress: A daily examination of temporal relationships in women migraineurs. Headache $37,553-558$.

Hökerberg, Y.H., Aguiar, O.B., Reichenheim, M., Faerstein, E., Valente, J.G., Fonseca, M.J., Passos, S.R. (2010). Dimensional structure of the demand control support questionnaire: A Brazilian context. Int Arch Occup Environ Health 83, 407-416.

Hu, H.X., Markson, L.E., Lipton, R.B., Stewart, W.F., Berger, M.L. (1999). Burden of migraine in the United States: Disability and economic costs. Arch Int Med 159, 813-818.

Jardim, C.P., Lotufo, P.A., Benseñor, I.M. (2001). Validation of a simple questionnaire for migraine diagnosis for use in epidemiological research. Cephalalgia 21, 303-304.

Johnson, J.V., Hall, E.M. (1988). Job strain, work place social support and cardiovascular disease: A cross-sectional study of a random sample of the Swedish working population. Am J Public Health 78, 1336-1342.
Karasek, R.A. (1979). Job demand, job decision latitude, and mental strain: Implications for job redesign. Adm Sci Q 24, 285-308.

Kelman, L. (2007). The triggers or precipitants of the acute migraine attack. Cephalalgia 27, 394-402.

Kivimäki, M., Nyberg, S.T., Batty, G.D., Fransson, E.I., Heikklä, K., Alfredson, L., Bjorner, J.B., Borritz, M., Burr, H., Casini, A., Clays, E., De Bacquer, D., Dragano, N., Ferrie, J.E., Geuskens, G.A., Goldberg, M., Hamer, M., Hooftman, W.E., Houtman, I.L., Joensuu, M., Jokela, M., Kittel, F., Knutsson, A., Koskenvuo, M., Koskinen, A., Kouvonen, A., Kumari, M., Madsen, I.E., Marmot, M.G., Nielsen, M.L., Nordin, M., Oksanen, T., Pentti, J., Rugulies, R., Salo, P., Siegrist, J., Singh-Manoux, A., Suominen, S.B., Väänänen, A., Vahtera, J., Virtanen, M., Westerholm, P.J., Westerlund, H., Zins, M., Steptoe, A., Theorell, T., for the IPD-Work Consortium (2012). Job strain as a risk factor for coronary heart disease: A collaborative meta-analysis of individual participant data. Lancet 380, 1491-1497.

Kopec, J.A., Sayre, E.C. (2004). Work-related psychosocial factors and chronic pain: A prospective cohort study in Canadian workers. $J$ Occup Environ Med 46, 1263-1271.

Lipton, R.B., Liberman, J.N., Kolodner, K.B., Bigal, M.E., Dowson, A., Stewart, W.F. (2003). Migraine headache disability and health-related quality-of-life: A population-based case-control study from England. Cephalalgia 23, 441-450.

Lipton, R.B., Stewart, W.F., Celentano, D.D., Reed, M.L. (1992). Undiagnosed migraine headaches: A comparison of symptom-based and reported physician diagnosis. Arch Intern Med 152, 1273-1278.

Lipton, R.B., Stewart, W.F., Diamond, S., Diamond, M.L., Reed, M. (2001). Prevalence and burden of migraine in the United States: Data from the American Migraine Study II. Headache 41, 646-657.

Lopes, C.S., Arraya, R., Werneck, G.L., Chor, D., Faerstein, E. (2010). Job strain and other work conditions: Relationships with psychological distress among civil servants in Rio de Janeiro, Brazil. Soc Psychiatry Psychiatr Epidemiol 45, 345-354.

Loyola Filho, A.I., Uchoa, E., Guerra, H.L., Firmo, J.O.A., Lima-Costa M.F. (2002). Prevalence and factors associated with self-medication: The Bambuí health survey. Rev Saude Publica 36, 55-62.

Macedo, L.E.T., Chor, D., Andreozzi, V., Faerstein, E., Werneck, G.L., Lopes, C.S. (2007). Job stress and interruption of routine activities due to health problems, according to the Pró-Saúde Study. Cad Saude Publica 23, 2327-2336.

Mäki, K., Vähtera, J., Virtanen, M., Elovainio, M., Keltikangas-Järvinen, L., Kivimäki, M. (2007). Work stress and new-onset migraine in a female employee population. Cephalalgia 28, 18-25.

Misakian, A.L., Langer, R.D., Benseñor, I.M., Cook, N.R., Manson, J.E., Buring, J.E., Rexrode, K.M. (2003). Postmenopausal hormone therapy and migraine headache. J Womens Health (Larchmt) 12, 1027-1036.

R Core Team. (2012) R: A language and environment for statistical computing. R Foundation for Statistical Computing.

Rivera-Torres, P., Araque-Padilla, R.A., Montero-Simó, M.J. (2013). Job stress across gender: The importance of emotional and intellectual demands and social support in women. Int J Environ Res Public Health 10, 375-389.

Sauro, K.M., Becker, W.J. (2009). The stress and migraine interaction. Headache 49, 1378-1386.

Stewart, W.F., Ricci, J.A., Chee, E., Morganstein, D., Lipton, R. (2003). Lost productive time due to pain conditions in the US workforce. JAMA 290, 2443-2454.

Stewart, W.F., Wood, C., Reed, M.L., Roy, J., Lipton, R.B., on the behalf of the AMPP Advisory Group (2008). Cumulative lifetime migraine incidence in women and men. Cephalalgia 28, 1170-1178.

The Headache Classification Subcommittee of the International Headache Society (2004). The international classification of headache disorders, 2nd ed. Cephalalgia 24, 1-151.

Wilkins, K., Beaudet, M.P. (1998). Work stress and health. Health Rep 10, $47-62$. 